The Nepali Math. Sc. Report

Vol.36, No.1 and 2, 2019

\title{
EFFICIENT ALGORITHM FOR MINIMUM COST FLOW PROBLEM WITH PARTIAL LANE REVERSALS
}

\author{
URMILA PYAKUREL \\ Central Department of Mathematics, Tribhuvan University, \\ PO Box 13143, Kathmandu, Nepal \\ urmilapyakurel@gmail.com
}

\begin{abstract}
In this paper, we investigate the minimum cost flow problem in two terminal series parallel network. We present modified minimum cost flow algorithm that computes the maximum dynamic and the earliest arrival flows in strongly polynomial time and also preserves all unused arc capacities. We also present strongly polynomial time minimum cost partial contraflow algorithm that solves both problems with partial reversals of arc capacities on two terminal series parallel networks.
\end{abstract}

Key Words: minimum cost flow, partial contraflow, efficient algorithm, complexity.

AMS (MOS) Subject Classification. Primary: 90B10, 90C27, 68Q25; Secondary: 90B06, $90 \mathrm{~B} 20$.

\section{INTRODUCTION}

After any kind of disasters, the process of saving lives by sending them in safe areas from the disastrous areas as quickly and efficiently as possible is considered as an evacuation planning problem. There are many terrible and remarkable disasters in the world, for example earthquakes in Nepal (1934 and April 2015), Japan (March 2011), Haiti (January 2010), Chichi (Taiwan, September 1999), Bam (Iran, December 2003), Kashmir (Pakistan, October 2005) and Chile (May 1960), various tsunamis in Japan and the Indian Ocean, the major hurricanes Katrina, Rita and Sandy in USA, and the September 11 attacks in USA. In literature, there are number of mathematical models presented to solve the evacuation planning problem [2].

We consider a dynamic network to represent the evacuation scenario. The disastrous zone is considered as the source and safe area is the sink. All intersections of streets in between these two nodes are considered as intermediate nodes. All connections among these nodes are the arcs. Each node and arc are bounded by capacities. Each arc has a transit time or a cost function. Group of evacuees that passes through the network over time is modeled as the flow.

Following the pioneer foundation work of Ford and Fulkerson [4] with an objective of maximizing the flow from a source to a sink at the end of given discrete time period, Gale [5] shows an existence of the maximum flow from the very beginning in discrete time setting,

Received December 1, 2019 
i.e. earliest arrival flow. To solve the earliest arrival flow problem, two pseudo-polynomial time algorithms are presented by Wilkinson [19] and Minieka [7]. The arc transit times are taken to be constant. Later Ruzika et al. [18] have presented strongly polynomial time algorithm to solve the earliest arrival flow problem on two terminal series parallel (TTSP) network.

During the evacuation process, the emergency planners discourage people to move towards the risk areas from the safer places because of which the corresponding road lanes are unoccupied. However, the lanes directing outwards from sources become more congested due to large number of evacuees and vehicles on the streets. The optimal lane reversal strategy makes the traffic systematic and smooth by removing the traffic jams caused in different large scale natural and man-made disasters, busy office hours, special events and street demonstrations. The contraflow reconfiguration, by means of various operations research models, different heuristics, optimization and simulation techniques reverses the usual direction of empty lanes towards the sinks that satisfy the given constraints, increase the flow value and decrease the average evacuation time. Authors in $[6,17,1,2,8,10$, $11,12,13,14,15]$ study various contraflow evacuation planning problems and presented different models with efficient algorithms for their solutions.

In this paper, we modify the minimum cost flow algorithm of Ruzika et al. [18] and obtain the earliest arrival flow on TTSP-network by saving all unused arc capacities. Moreover, we present the minimum cost partial contraflow algorithm on two terminal series parallel network for the first time. These both algorithms are solvable in polynomial time complexity.

The organization of the paper is as follows. Section 2 states the basic concepts and flow models. Section 3 investigates the minimum cost flow problem solves the earliest arrival flow problem and presents a modified algorithm. In Section 4, the minimum cost flow for contraflow problem is investigated and is presented a strongly polynomial time algorithm to solve the maximum dynamic and the earliest arrival flow problems with partial lane reversals. Section 5 concludes the paper.

\section{Preliminaries}

Consider an evacuation network $\mathcal{N}=(V, A, b, \tau, s, d, T)$ with set of nodes $V$, set of arcs $A$, arc capacity function $b: A \rightarrow \mathbb{Z}_{\geq 0}$, arc travel time function $\tau: A \rightarrow \mathbb{Z}_{\geq 0}$, single source $s \in V$, single sink $d \in V$ and given integer time horizon $T$. The capacity of an arc limits the amount of flow traveling on the arc. Travel time represents the total time needed for the flow to travel on the arc. We have finite number of nodes and arcs, i.e., $|V|=n$ and $|A|=m$. We assume that there is no any incoming arc to the source and outgoing arc from the sink. Let $A_{i}=\{(i, j) \in A: j \in V\}$ and $B_{i}=\{(j, i) \in A: j \in V\}$ be the set of outgoing arc and incoming arcs for node $i$, respectively. So that without contraflow, we have $A_{d}=B_{s}=\emptyset$.

A static $s-d$ flow $x: A \rightarrow \mathbb{R}_{\geq 0}$ of value $\operatorname{val}(x)$ in objective function (2.1) satisfies the flow conservation and capacity constraints (2.2) and (2.3), respectively, [4] 


$$
\begin{aligned}
\operatorname{val}(x)=\sum_{(i, j) \in B_{d}} x(i, j) & =\sum_{(i, j) \in A_{s}} x(i, j) \\
\sum_{(i, j) \in B_{v}} x(i, j)-\sum_{(i, j) \in A_{v}} x(i, j) & =0, \quad \forall v \in V \backslash\{s, d\} \\
b(i, j) \geq x(i, j) & \geq 0, \quad \forall(i, j) \in A
\end{aligned}
$$

The maximum static flow maximizes the objective function (2.1) subject to (2.2) and (2.3). Constraint (2.2), called the flow conservation constraint, indicates that total inflow into a node $v$ must be equal to total outflow from it. These constraints imply that the total inflow into sink $d$ must be equal to the total outflow from source $s$. Feasibility of the flow is bounded by the capacity constraint $(2.3)$, i.e., flow $x(i, j)$ can not be greater than the capacity of an $\operatorname{arc}(i, j)$ for all $(i, j) \in A$. If the flow conservation constraint is also posed at $s$ and $d$, then a circulation is obtained.

If the unit costs along the arcs are also considered then we have to minimize the total cost. If we are given a flow value $\operatorname{val}(x)$, the minimum cost flow problem seeks to shift the flow with minimum cost $\sum_{(i, j) \in A} c(i, j) x(i, j)$ subject to $(2.2)$ and (2.3) and $\sum_{(i, j) \in B_{d}} x(i, j)=\operatorname{val}(x)$. The minimum cost flow problem with zero circulation turns into the minimum cost circulation flow problem.

Let $y: A \times \mathbf{T} \rightarrow R^{+}$be a dynamic flow in discrete time $\mathbf{T}$. The dynamic flow maximizes $\operatorname{val}(y, T)$ in the objective function (2.4) subject to the constraints (2.5-2.7) without intermediate storage in time $T$, although, according to constraint (2.6), some flow may wait in intermediate notes at time $\theta<T[3,4]$.

$$
\begin{aligned}
\sum_{(i, j) \in A_{s}} \sum_{\sigma=0}^{T} y(i, j)(\sigma) & =\sum_{(i, j) \in B_{d}} \sum_{\sigma=\tau(i, j)}^{T} y(i, j)(\sigma-\tau(i, j)) \\
\sum_{(i, j) \in B_{v}} \sum_{\sigma=\tau(i, j)}^{T} y(i, j)(\sigma-\tau(i, j)) & =\sum_{(i, j) \in A_{v}} \sum_{\sigma=0}^{T} y(i, j)(\sigma), \forall v \notin\{s, d\} \\
\sum_{(i, j) \in B_{v}} \sum_{\sigma=\tau(i, j)}^{\theta} y(i, j)(\sigma-\tau(i, j)) & \geq \sum_{(i, j) \in A_{v}} \sum_{\sigma=0}^{\theta} y(i, j)(\sigma), \forall v \notin\{s, d\}, \theta \in \mathbf{T} \\
b(i, j)(\theta) \geq y(i, j)(\theta) & \geq 0, \forall(i, j) \in A, \theta \in \mathbf{T}
\end{aligned}
$$

If the objective is to maximize the flow at every time from the beginning, we call it the earliest arrival flow problem. The objective function of the earliest arrival flow is (2.8) which is to be maximized at every time $\theta$ subject to the constraints (2.5-2.7).

$$
\operatorname{val}(y, \theta)=\sum_{\sigma=0}^{\theta} \sum_{(i, j) \in A_{s}} y(i, j)(\sigma)=\sum_{\sigma=\tau(i, j)}^{\theta} \sum_{(i, j) \in B_{d}} y(i, j)(\sigma-\tau(i, j)) .
$$




\section{Modified minimum COST Flow ALgORIthm}

In this section, we present a modified minimum cost flow algorithm on TTSP network that solves the maximum dynamic and the earliest arrival flow problems in strongly polynomial time complexity and records all the unused arc capacities of the network simultaneously.

The earliest arrival flow problem finds the maximum dynamic flow at every point of time from the beginning. In general network, the problem is $N P$-hard. Even in two terminal network, the problem is not solved in polynomial time. Authors in $[7,19]$ presented pseudopolynomial algorithms to solve the problem in general two terminal network by computing the successive shortest paths in time expanded network. If the network is reduced to TTSP network, then, a strongly polynomial time algorithm has been presented to solve the problem by sending the flow iteratively through the $s$ - $d$ paths with the minimum time and removing the saturated arcs. Only the paths within time horizon $T$ is taken in this greedy approach (Ruzika et al. [18]).

Here, we modify the greedy algorithm of Ruzika et al. [18] in which we record the residual capacity at each time point. If there is no any augmenting path in the residual network, then the flow is maximum, [3]. We collect the residual capacity that is not used by the augmenting path as unused or saved capacity $b_{s c a}$. With this modification on minimum cost flow algorithm of Ruzika et al. [18], we present Algorithm 1. First we convert the $s-d$ network into circulation by adding an arc $(d, s)$ with infinite capacity and $-(T+1)$ cost where transit time on each arc is considered as cost. So that we have a new set of arcs $A=A \cup\{(d, s)\}$. Then, we solve the minimum cost circulation problem. For the maximum flow, the flow along arc $(d, s)$ is ignored and the resulting $s-d$ flow is decomposed into $s-d$ paths $P_{k}, m \geq k \geq 1$. At each time point, a static $s$ - $d$ flow value along each path $P_{k}$ is computed iteratively, and this process should be terminated within the estimated time horizon $T$. Moreover, we record all the unused arc capacities $b_{s c a}=b(i, j)-x(i, j)$ from all unsaturated arcs.

Theorem 3.1. The temporally repeated flow computation of static path flow obtained in Algorithm 1 within time $T$ gives the maximum dynamic flow and the earliest arrival flows by saving all unused arc capacities on TTSP network.

Proof. Algorithm 1 computes the cheapest path $P_{k}, 1 \leq k \leq m$ with path cost $\tau\left(P_{k}\right)=$ $\sum_{(i, j) \in P_{k}} \tau(i, j)$, assigns flow value $x\left(P_{k}\right)$ to it, records the residual capacities $r_{c a}(i, j)$ of each arc $(i, j) \in P_{k}$ and terminates with $\tau\left(P_{k}\right) \geq T+1$. If there is no any $s$ - $d$ path within $T$, the maximum static flow $x=\sum_{P_{k} \in \mathcal{P}_{k}} x\left(P_{k}\right)$ is obtained and the unsaturated arc capacities $b_{s c a}$ is recorded.

The maximum dynamic flow $y$ is computed with temporally repeated flow with cheapest paths $P_{k}$ and path flow $x\left(P_{k}\right)$ as follows

$$
\operatorname{val}(y, T)=\sum_{P_{k} \in \mathcal{P}}\left(T-\tau\left(P_{k}\right)+1\right) x\left(P_{k}\right)
$$




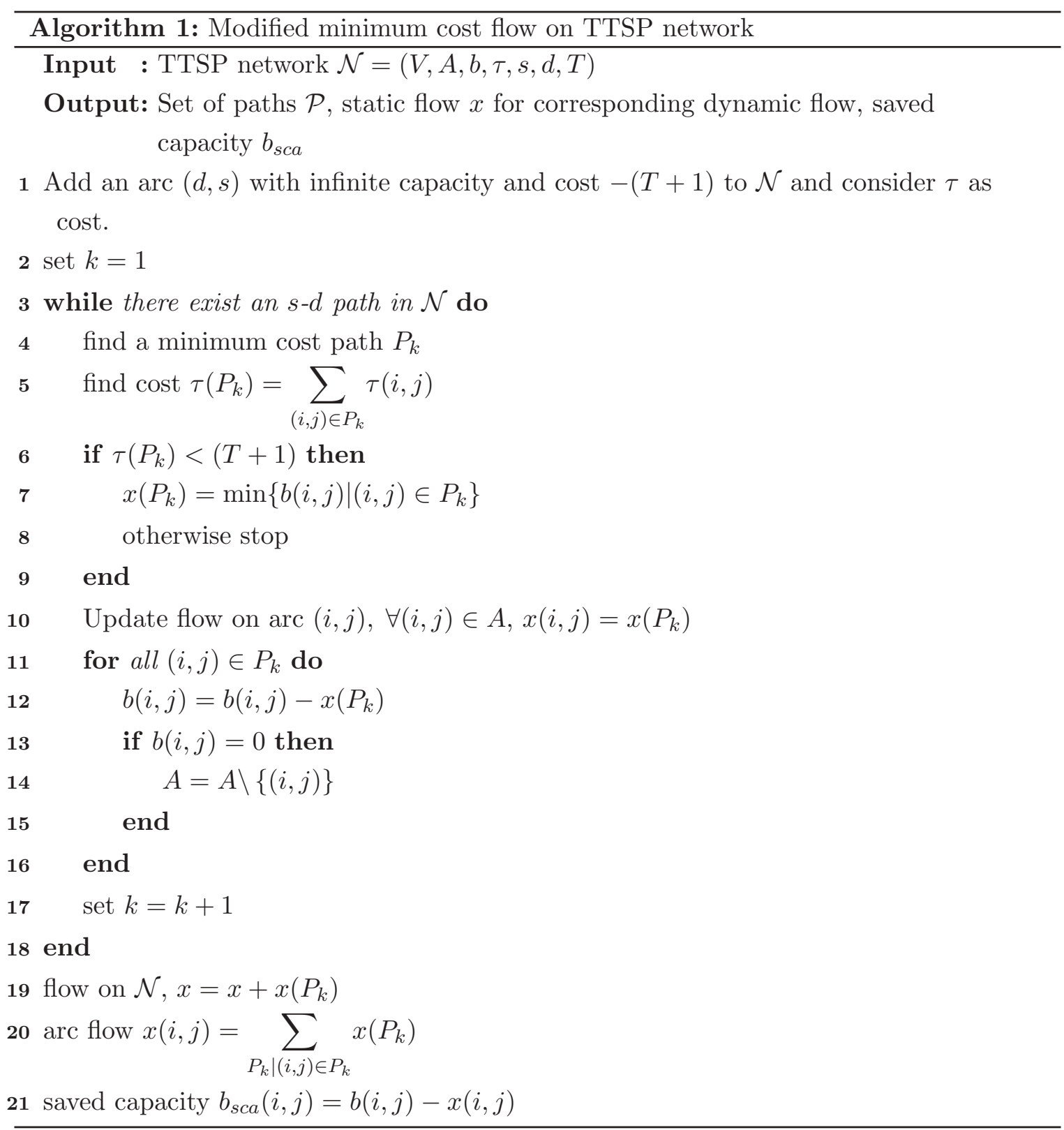

As the obtained flow satisfy the earliest arrival property, i.e., a cumulative amount of flows reaching the sink, in every considered time period and all preceding time periods of the considered one, has to be maximal.

Corollary 3.2. The earliest arrival flow on TTSP network can be computed by recording all unused arc capacities in strongly polynomial time complexity.

Proof. The minimum cost flow problem on TTSP network is solved by computing successive shortest paths in which each path cost should not exceed the time horizon $T$ in time $O(m n+$ $m \log m$ ), (Ruzika et al. [18]). The unused arc capacities are recored in constant time. Thus, the time complexity of Algorithm 1 is $O(m n+m \log m)$.

Example 3.3. Figure 1(a) represents the TTSP network. Each arc contains capacity and transit time. Before contraflow configuration of the network, the earliest arrival flow value 
in time horizon $T=6$ obtained by solving with Algorithm 1 is 16 via paths $P_{1}=s-x-u-d$, $P_{1}=s-y-w-d$ and $P_{3}=s-x-v-d$. The unused arc capacities are $[s, y: 1],[y, w ; 1]$ and $[v, d ; 3]$.
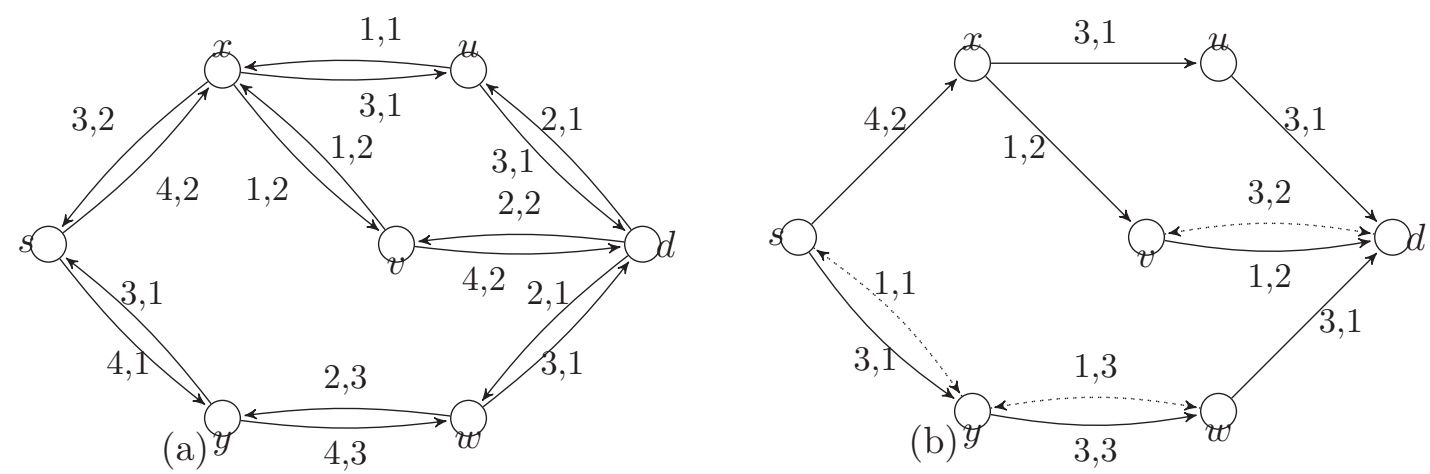

Figure 1. EAPCF on two terminal series parallel graph

\section{Minimum cost flow partial CONTRAflow Algorithm}

In this section, we present an efficient minimum cost flow algorithm to solve the partial contraflow problem. Recently, author in $[15,16]$ introduced the partial contraflow approach for evacuation planning and investigated different solution algorithms to solve the problem for constant and inflow dependent transit times on each arcs.

In partial contraflow approach, we allow the reversal of arc capacities only the necessary portion. This approach has great benefit as the remaining unused arc capacities can be used for emergency vehicles and logistics. With the minimum cost flow algorithm, we solve the maximum dynamic as well as the earliest arrival flow problems with partial lane reversals in TTSP network by temporal repetition of path flows and saving all unused arc capacities.

Theorem 4.1. The minimum cost partial contraflow always gives the maximum dynamic contraflow on TTSP network with partial reversals of lanes.

Proof. All the lines of our algorithm except while loop of lines (8-13) are feasible. As the flow obtained in line 7 does not contains any cycle with positive flow, both arcs $(i, j)$ and $(j, i)$ cannot be used simultaneously. Therefore, the flow in auxiliary network cannot greater than the reversed capacity of the arcs. This shows that Algorithm 2 is feasible.

As in previous works $[1,9,16]$, the minimum cost flow gives the maximum dynamic flow with temporally repeated paths flows on auxiliary network as in Theorem 3.1 and the solution is equivalent to the maximum dynamic flow with lane reversals on original network. However, in our algorithm, we compute residual arc capacities on each time step so that we can reverse only the necessary part of the arcs and use the remaining arc capacities for other security purpose. Thus, our algorithm computes the optimal maximum dynamic contraflow in the same complexity by saving all unused arc capacities.

Theorem 4.2. Algorithm 2 gives the earliest arrival flow with partial lane reversals on TTSP network. 


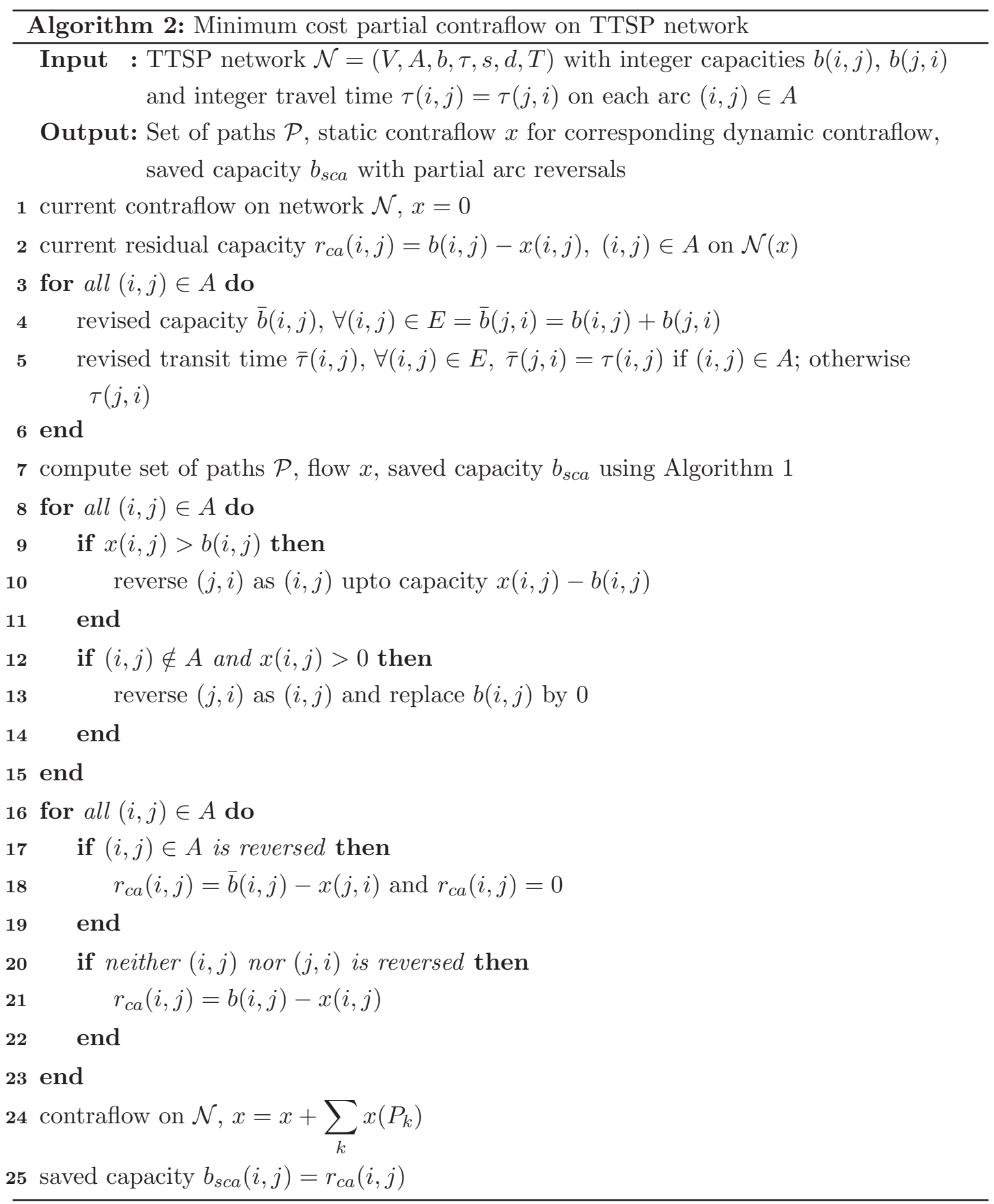

Proof. The maximum dynamic flow with partial lane reversals is maximum for each time step from the beginning on TTSP network. Thus, Algorithm 2 gives the earliest arrival flow with partial lane reversals on TTSP network in polynomial time complexity $O(m n+$ $m \log m)$.

Example 4.3. We apply Algorithm 2 on Figure 1(a) to find the earliest arrival partial contraflow. First we construct the auxiliary network with contraflow configuration as in Steps (3-6) of Algorithm 2, see Figure 2(a). In the auxiliary network, we solve the modified minimum cost flow algorithm (cf. Algorithm 1) iteratively. As in Example 3.3, path $P_{1}=$ 
$s-x-u-d$ carries flow value 4 at times 4,5 and 6 . Path $P_{2}=s-y-w-d$ carries 5 flow value at time 5 and 6 . Path $P_{3}=s-x-v-d$ carries 2 flow value at time 6 . Thus in time $T=6$, the total flow 24 units is sent from $s$ to $d$ and unused arc capacities are recorded as $[s, x ; 1],[s, y ; 2],[y, w ; 1],[u, d ; 1],[v, d ; 4]$ in Figure $2(\mathrm{~b})$. As the flow is maximized at each time point by reversing only necessary arc capacities, it is the earliest arrival partial contraflow for given TTSP network of Figure 1(a).
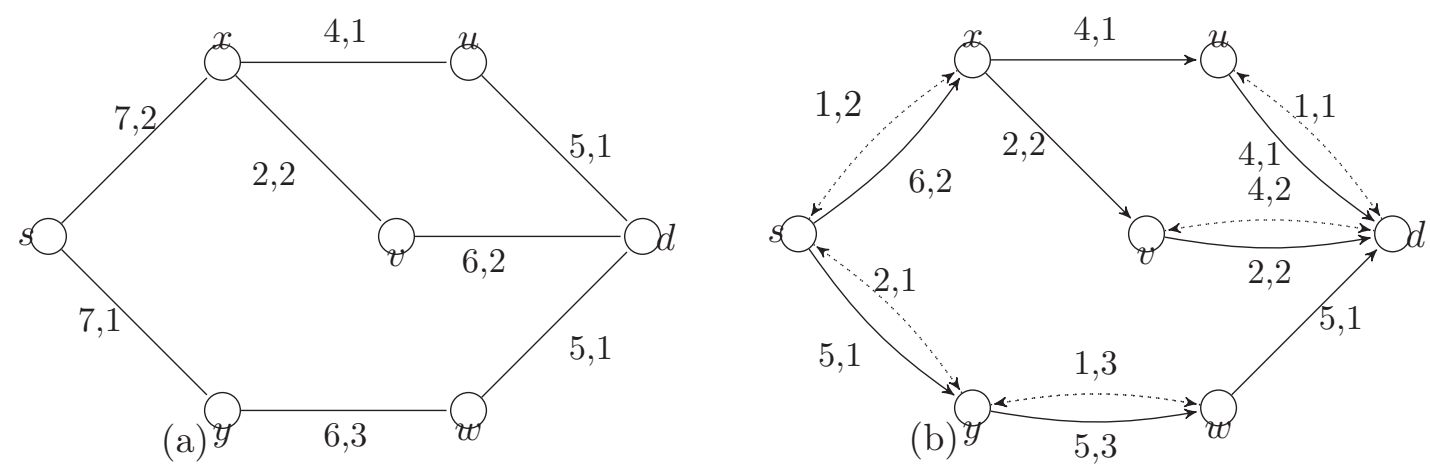

FiguRE 2. (a) Auxiliary network of Figure 1(a), (b) Earliest arrival partial contraflow

\section{Conclusions}

In this work, we studied the minimum cost flow problem and investigated its solution technique required to solve the dynamic flow problems on TTSP networks. We presented modified minimum cost flow algorithm that not only maximizes the flow along paths but also records all unused arc capacities of each arc on the network. Using this algorithm, we have solved the maximum dynamic and the earliest arrival flow problems on TTSP networks in strongly polynomial time complexities. Moreover, we presented the minimum cost partial contraflow algorithm that solves both maximum dynamic and earliest arrival flow problems with partial reversals of arc capacities. This algorithm has strongly polynomial time complexity.

\section{ACKNOWLEDGMENTS}

The author would like to thank Alexander von Humboldt Foundation for the Return Fellowship (November 2019-October 2020).

\section{REFERENCES}

[1] Dhamala, T.N. and Pyakurel, U. (2013). Earliest arrival contraflow problem on series-parallel graphs. International Journal of Operations Research, 10, 1-13

[2] Dhamala, T.N. Pyakurel, U. and Dempe, S. (2018). A critical survey on the network optimization algorithms for evacuation planning problems. International Journal of Operations Research, 15(3), 101133.

[3] Ford, F.R. and Fulkerson, D.R. (1958). Constructing maximal dynamic flows from static flows. Operations Research, 6, 419-433.

[4] Ford, L.R.; Fulkerson, D.R. Flows in Networks. Princeton University Press: Princeton, New Jersey, USA, 1962. 
[5] Gale, D. (1959). Transient Flows in Network . Michigan Mathematical Journal, Vol. 6, pp. 59-63.

[6] Kim, S.; Shekhar, S.; Min, M. Contraflow transportation network reconfiguration for evacuation route planning. IEEE Transactions on Knowledge and Data Engineering, 2008, 20, 1-15.

[7] Minieka, E. (1973). Maximal, lexicographic, and dynamic network flows. Operations Research, 21, 517527.

[8] Pyakurel U. (2016). Evacuation planning problem with contraflow approach. PhD Thesis, IOST, Tribhuvan University, Nepal.

[9] Pyakurel, U. and Dhamala, T.N. (2015). Models and algorithms on contraflow evacuation planning network problems. International Journal of Operations Research, 12, 36-46.

[10] Pyakurel, U. and Dhamala, T.N. (2016). Continuous time dynamic contraflow models and algorithms. Advances in Operations Research - Hindawi; Article ID 368587, 1-7.

[11] Pyakurel, U. and Dhamala, T.N. (2017a). Evacuation planning by earliest arrival contraflow, Journal of Industrial and Management Optimization 13, 489-503.

[12] Pyakurel, U. and Dhamala, T.N. (2017b). Continuous dynamic contraflow approach for evacuation planning. Annals of Operation Research, 253, 573-598.

[13] Pyakurel, U., Dhamala, T.N. and Dempe, S. (2017a). Efficient continuous contraflow algorithms for evacuation planning problems. Annals of Operations Research (ANOR), 254, 335-364.

[14] Pyakurel, U. Nath, H.N. and Dhamala, T.N. (2018). Efficient contraflow algorithms for quickest evacuation planning. Science China Mathematics, 61(11), 2079-2100.

[15] Pyakurel, U. Nath, H.N. and Dhamala, T.N. (2018). Partial contraflow with path reversals for evacuation planning. Annals of Operations Research, DOI: 10.1007/s10479-018-3031-8.

[16] Pyakurel, U. Nath, H.N., Dempe, S. and Dhamala, T.N. (2019). Efficient Dynamic Flow Algorithms for Evacuation Planning Problems with Partial Lane Reversal. Mathematics, 7(10), 993. https : //doi.org/10.3390/math7100993

[17] Rebennack, S.; Arulselvan, A.; Elefteriadou, L.; Pardalos, P.M. Complexity analysis for maximum flow problems with arc reversals. Journal of Combinatorial Optimization 2010, 19, 200-216.

[18] Ruzika, S. Sperber, H. and Steiner, M. (2011). Earliest arrival flows on series-parallel graphs. Networks, $\mathbf{5 7}(2), 169-173$.

[19] Wilkinson, W.L. (1971). An algorithm for universal maximal dynamic flows in a network. Operations Research, 19: 1602-1612. 
